CLINICAL ETHICS

\title{
Authorship ignorance: views of researchers in French clinical settings
}

\section{B Pignatelli, H Maisonneuve, F Chapuis}

J Med Ethics 2005;31:578-581. doi: 10.1136/jme.2004.009449

See end of article for authors' affiliations

.....................

Correspondence to: Hervé Maisonneuve, Public Health Department, Hôpital Fernand Widal, 200 rue du Fg St Denis, 75010 Paris, France; hervemaison@wanadoo.fr

Received 24 May 2004 In revised form 30 December 2004 Accepted for publication 11 January 2005

\begin{abstract}
Objectives: To assess the knowledge and behaviour of researchers regarding criteria for authorship, and the practices of ghost and gift authorship.

Design: Semidirective interviews of senior clinical researchers.

Setting: University hospital.

Participants: Thirty-nine main investigators of clinical research programmes.

Main measurements: Awareness and use of International Committee of Medical Journal Editors (ICMJE) criteria for authorship, and perceptions about ghost and gift authorship.

Results: A total of 48 protocols submitted by 42 principal investigators between 1994 and 1996 were identified. Thirty-nine investigators were contacted; 37 (one of whom delegated a co-author) were interviewed between May 2002 and March 2003. Two co-authors of two principal investigators were also interviewed. In all, 42 studies were represented. The interviews lasted for 40-90 minutes and were conducted with openness and respect for confidentiality.

The choice of names of co-authors did not follow the ICMJE recommendations. Half of the respondents stated they were aware of criteria for authorship and knew of ICMJE, but most of them did not cite any of the ICMJE criteria among those they applied in deciding authorship. Most of them disagreed with the obligation to meet the three criteria justifying co-authorship because they found these too rigid and inapplicable. Gift authorship was a common practice; $59 \%$ of the respondents had been a recipient of gift authorship. Twenty-five (64\%) were aware of ghost authorship and the majority considered it questionable and blameworthy.

Conclusions: The ICMJE criteria were ignored by clinicians at a university hospital. Ghost and gift authorship were frequent among them. There is a need for French guidelines for authorship to be prepared and implemented.
\end{abstract}

$\mathrm{D}$ efinitions of authorship and the behaviour of authors vary in different countries and among specialties. Criteria such as those of the International Committee of Medical Journal Editors (ICMJE; http://www.icmje.org/) were first published in 1978. These uniform requirements have been regularly updated (last update October 2004), but the authorship criteria do not seem to have been responsible for much change in authors' behaviour. ${ }^{1}$ The complexities of academic promotion, based largely on the number of publications rather than on the quality of articles, contributes to the opacity of the way in which authorship is attributed. Confidence should be the basis of the system but the rules and guidelines do not seem to be known by authors. ${ }^{2}$ False authorship is a concern of most editors of prestigious journals, but editors can have little influence on the behaviour of scientists. ${ }^{3}$ Questions of authorship are debated at meetings such as the peer review congresses, ${ }^{5}$ but research is difficult because authors do not like to disclose their practices. A survey among 66 staff from a British medical faculty studied their awareness and use of criteria for authorship, and their experience of authorship strategies. Fifty respondents supported criteria for authorship but few knew about or used those that were available. ${ }^{1}$ Gift authorship was perceived as common. A difference was observed between editors' criteria and researchers' practice.

Most studies concerning this question have been conducted in English speaking countries. Only a few results from other countries have been presented. Such reports include a survey of authors of articles published in the Chinese Medical Journal, the Chinese Journal of Neurology, and the Chinese Journal of Pediatrics, which showed that 197 of 524 (38\%) authors met the ICMJE criteria. ${ }^{6}$ A study of authors submitting articles to Revista Medica de Chile showed low compliance with the criteria of authorship. ${ }^{7}$ In France, authorship has not been investigated, and researchers and clinicians have a poor understanding of authorship criteria; they follow the advice of senior professionals. The topic is rarely discussed in French biomedical journals, but there is informal consensus that problems are encountered everywhere.

We conducted a study of current practices in the preparation and publication of clinical research studies. We sought to ascertain researchers' behaviour during the publishing process and learn their views on the authorship of biomedical articles.

\section{METHODS}

We selected all clinical research programmes that were financed by the Projets Hospitaliers de Recherche Clinique (national funding from the Ministry of Health) for research teams at the Lyons public hospitals in 1994, 1995, and 1996. The main investigator of each programme received a letter (dated 11 May 2002), signed by the Chairman of the Scientific Committee of Lyons Hospitals (Professor L Collet, MD), introducing the study and asking for a 30-minute interview. Telephone follow-up was carried out to arrange an appointment. Another letter was sent on 25 October 2002 to the main investigators who had not arranged appointments by that date.

Abbreviation: ICMJE, International Committee of Medical Journal Editors 
Table 1 Quality of the 39 interviews on a four-digit scale assigned by the interviewer at the end of each meeting

\begin{tabular}{lllll}
\hline & Poor & Average & Good & Very good \\
\hline Clearness and spontaneity of answers & 2 & 7 & 7 & 23 \\
Cooperation during the interview & 5 & 5 & 13 & 16 \\
Sympathy and openness & 2 & 9 & 16 & 12 \\
\hline
\end{tabular}

Semidirective interviews were conducted by one of the authors (BP) at the interviewees' workplace. An introductory statement was provided to gain the confidence of the participants, and confidentiality was agreed orally before starting the interview. A questionnaire in French (available from the authors on request) was used and yes/no answers were obtained during the interview and reported on a transcript. Handwritten comments were added when the interviewees were talkative.

All answers were tabulated and the data were analysed using the software program SPSS (http://www.apss.com).

\section{RESULTS}

A total of 48 protocols submitted by 42 principal investigators between 1994 and 1996 were identified. Thirty-nine investigators were contacted; 37 (one of whom delegated a coauthor) were interviewed between May 2002 and March 2003 (representing 42 studies); two co-authors of two principal investigators were also interviewed. The investigators were aged $52 \pm 8$ years on average (range 38-69); 16 were aged $\leqslant 50$ years $(44 \pm 3)$ and 23 were aged between 51 and 69 years $(58 \pm 4)$. Seventeen were heads of clinical units, 10 were heads of both clinical and research units, five were hospital clinicians, six were hospital clinicians and leaders of research units, and one was a scientist leading a research team. Twenty-three (59\%) of the investigators had published between five and 100 articles, and 16 (41\%) had published between 101 and 400 articles. The interviews lasted for $40-$ 90 minutes; most of them were conducted with openness (table 1) and confidentiality was respected. Twenty-two of the 39 interviewees expressed a spontaneous interest in the study.

Of the 42 protocols, one was never performed and six were not published; four had been submitted for publication at the time of the interview, and 31 had been published by 28 principal investigators. We obtained more information about 29 of the 31 published articles: one was accepted without modification, 20 were accepted after the first resubmission, 18 took into account all remarks from referees, and complementary data were added to 11 . For the 31 published studies, all 28 principal investigators declared that they had participated in the study conception and protocol design; 22 had had a role in the literature search; 27 had participated in reading and interpreting the literature; 11 had participated in the data gathering, 13 in the data analysis, and 27 in the interpretation of the collected data; five gave advice on the statistical analysis. Fourteen of the 28 principal investigators had participated in writing the first draft, 17 declared they had written parts of the article, 27 had critically assessed the article, and all had checked the final version and approved the final draft. Nineteen were corresponding authors, 26 signed the submission form declaring that they had participated in the study, and 21 had read the galley proofs.

Most often the choice of names of co-authors did not follow the ICMJE recommendations (table 2). No investigator reported that the journal publisher queried the number or order of the authors. Various procedures were described and applied by the investigators: a decision was made by one or two authors (the first and last authors of the article, including the head of a clinical unit, who was always given as the first or the last author), or by a group of authors, or according to hierarchy. Predetermined rules were used, such as the inclusion of one person from each participating centre or discipline (ordered by number of recruited patients). Authorship policies based on implicit criteria for promotion and career prospects had been customised over time by the academic senior clinicians; they were so diverse that we did not identify any particular cluster. Half of the respondents stated that they were aware of criteria for authorship and that they had heard of the ICMJE, but most of them did not know the ICMJE criteria and did not cite any of them among the criteria they had applied (tables 2 and 3). Most researchers said they agreed with the first two criteria. Most disagreed with the obligation to fulfil the three criteria justifying co-authorship because they found the conditions too rigid and irrelevant. They mentioned that the participation of some essential collaborators, such as those who recruited patients, ought to be acknowledged.

Gift authorship was a common practice (table 4); $59 \%$ of the respondents considered it questionable and blameworthy. Agreement with each of the four proposals offered for reducing gift authorship was variable, but almost all of the researchers thought that these proposals were ineffectual. Among the most frequent reasons given for gift authorship were: pressure to publish; the repayment or exchange of favours; the search for a scientific guarantee that would enhance the chances of publication; thanks (not linked to the publication) to scientific seniors; the maintenance of good relationships; establishing a good record to begin or to develop a career as well as to obtain nominations or promotions; and traditional habits such as systematically granting authorship to the head of a clinical unit.

Table 2 Process for choosing co-authors, as expressed by the 28 principal investigators who participated in writing the articles

\begin{tabular}{lrr}
\hline & Number & $\%$ \\
\hline Choice of co-authors & 6 & 21 \\
Solely by the main author & 5 & 18 \\
After consultation with co-authors & 1 & 4 \\
On request of co-authors & 16 & 57 \\
Another way & & \\
Use of criteria & 25 & 89 \\
Yes & 3 & 11 \\
No & 4 & 14 \\
Use of one of the ICMJE criteria & 24 & 86 \\
Yes & 20 & 71 \\
No & 8 & 29 \\
Intervention of co-authors & & \\
Yes & 5 & 18 \\
No & 5 & 0 \\
Order of co-authors & 0 & 64 \\
Solely by the main author & 18 & \\
After consultation with co-authors & \\
On request of co-author & & \\
Another way &
\end{tabular}


Table 3 Criteria for authorship expressed by the 39 interviewees

\begin{tabular}{|c|c|c|}
\hline & Number & $\%$ \\
\hline \multicolumn{3}{|c|}{ Do you know the criteria for authorship? } \\
\hline Yes & 20 & 51 \\
\hline No & 19 & 49 \\
\hline \multicolumn{3}{|c|}{ ICMJE criteria cited among applied criteria } \\
\hline None & 33 & 84 \\
\hline One & 3 & 8 \\
\hline More than one & 3 & 8 \\
\hline \multicolumn{3}{|c|}{ Do you know about ICMJE? } \\
\hline Yes & 20 & 51 \\
\hline No & 19 & 49 \\
\hline \multicolumn{3}{|c|}{ Do you know the ICMJE criteria? } \\
\hline Yes & 2 & 5 \\
\hline \multirow{2}{*}{\multicolumn{3}{|c|}{$\begin{array}{l}\text { What is your opinion of the } 3 \text { ICMJE criteria? } \\
\text { (1) Substantial contribution to conception and } \\
\text { design, or acquisition of data, or analysis and } \\
\text { interpretation of data }\end{array}$}} \\
\hline & & \\
\hline Agree & 38 & 97 \\
\hline Disagree & 1 & 3 \\
\hline \multicolumn{3}{|c|}{$\begin{array}{l}\text { (2) Drafting the article or revising it critically for } \\
\text { important intellectual content }\end{array}$} \\
\hline Agree & 31 & 80 \\
\hline Disagree & 6 & 15 \\
\hline Don't know & 2 & 5 \\
\hline \multicolumn{3}{|c|}{ (3) Final approval of the version to be published } \\
\hline Agree & 24 & 62 \\
\hline Disagree & 13 & 33 \\
\hline Don't know & 2 & 5 \\
\hline \multicolumn{3}{|c|}{ The 3 criteria must all be met } \\
\hline Agree & 7 & 18 \\
\hline Disagree & 30 & 77 \\
\hline Don't know & 2 & 5 \\
\hline \multicolumn{3}{|c|}{ Are ICMJE criteria applied? } \\
\hline Yes & 16 & 41 \\
\hline No & 14 & 36 \\
\hline Don't know & 9 & 23 \\
\hline
\end{tabular}

Twenty-five (64\%) respondents were aware of ghost authorship and the majority considered it questionable and blameworthy (table 5). Its frequency was regarded as far from negligible and the interviewees stated that they had often received it or allowed it themselves. The researchers identified three major sources of ghost authorship: authoritarianism prevailing in some teams and concomitant disrespect; dishonest appropriation or lack of recognition of work; and relational, scientific or interest conflicts. The frequent occurrence of gift and ghost authorship is also shown by the data in table 6 .

\section{DISCUSSION}

For the first time in France researchers have been interviewed on their publication practices; this has shown that the ICMJE criteria are being ignored. Ghost and gift authorship are frequent. There is a need for French guidelines on authorship to be proposed and implemented.

\section{Quality of the study}

The interviews lasted for more than the 20-30 minutes planned and their quality showed the great interest of the respondents in the subject of this study. All the respondents were senior researchers and agreed to share their experiences openly. These topics are not usually discussed and their anonymity was necessary. These researchers agreed to give their opinions on authorship behind closed doors. We preferred that interviews should be conducted by the same person, even if, as a result, the sample of respondents had to be smaller. The quality of the discussions and the atmosphere of confidence established between the interviewer and the respondents allowed more accurate data to be obtained than
Table 4 Gift authorship expressed by the 39 interviewees

\begin{tabular}{|c|c|c|}
\hline & Number & $\%$ \\
\hline \multicolumn{3}{|c|}{ Gift authorship is questionable } \\
\hline Agree & 23 & 59 \\
\hline Disagree & 8 & 20 \\
\hline Don't know & 8 & 20 \\
\hline \multicolumn{3}{|c|}{ Frequency of gift authorship } \\
\hline Rare & 5 & 13 \\
\hline Frequent & 16 & 41 \\
\hline Very frequent & 14 & 36 \\
\hline Don't know & 4 & 10 \\
\hline \multicolumn{3}{|c|}{ Gift authorship must be condemned } \\
\hline Agree & 22 & 56 \\
\hline Disagree & 11 & 28 \\
\hline Don't know & 6 & 16 \\
\hline \multicolumn{3}{|c|}{$\begin{array}{l}\text { How can gift authorship be reduced? } \\
\text { (1) Declaration signed by the authors }\end{array}$} \\
\hline Agree & 27 & 69 \\
\hline Disagree & 12 & 31 \\
\hline Don't know & 0 & 0 \\
\hline \multicolumn{3}{|c|}{$\begin{array}{l}\text { (2) Limit the number of publications on a CV } \\
\text { sent with a job application }\end{array}$} \\
\hline Agree & 14 & 36 \\
\hline Disagree & 24 & 61 \\
\hline Don't know & 1 & 3 \\
\hline \multicolumn{3}{|c|}{ (3) Credits shared among authors } \\
\hline Agree & 10 & 25 \\
\hline Disagree & 28 & 72 \\
\hline Don't know & 1 & 3 \\
\hline \multicolumn{3}{|c|}{ (4) Contributorship system } \\
\hline Agree & 20 & 51 \\
\hline Disagree & 18 & 46 \\
\hline Don't know & 1 & 3 \\
\hline
\end{tabular}

Table 5 Ghost authorship (familiar to 25 of the 39 interviewees)

\begin{tabular}{lrr}
\hline & Number & $\%$ \\
\hline Is ghost authorship questionable? & 23 & 92 \\
Yes & 0 & 0 \\
No & 2 & 8 \\
Don't know & 7 & 28 \\
What is the frequency of ghost authorship? & 8 & 32 \\
$\quad$ Rare & 2 & 8 \\
$\quad$ Frequent & 8 & 32 \\
Very frequent & 21 & 84 \\
Don't know & 0 & 0 \\
Should ghost authorship be condemned? & 4 & 16 \\
Yes & & \\
No & & \\
Don't know & 9 & 36 \\
Do you have any proposals for banning ghost & \\
authorship? & 16 & 64 \\
Yes & & \\
No & & \\
\hline
\end{tabular}

if we had mailed a questionnaire accompanied by a reply envelope. An open discussion after the questionnaire allowed various matters to be clarified with the participants. The questionnaire was based on previous ones used elsewhere. ${ }^{13} 8$ It was adapted to the French language and verified as suitable for French scientists. The completeness of the data obtained and the good understanding achieved with the interviewees confirmed us in our choice of an interview method.

The interviews were carried out during 2002 and at the beginning of 2003 for researchers who were funded between 1994 and 1996. This time lag was necessary to allow for time to publish the articles. Answers were based on memory, but 
Table 6 Experience as an author of articles expressed by the 39 interviewees

\begin{tabular}{|c|c|c|}
\hline & Number & $\%$ \\
\hline \multicolumn{3}{|c|}{$\begin{array}{l}\text { Have you discovered after the publication } \\
\text { of an article that you were one of its authors? }\end{array}$} \\
\hline Yes & 24 & 62 \\
\hline No & 15 & 38 \\
\hline \multicolumn{3}{|c|}{$\begin{array}{l}\text { Has your name been omitted from an article } \\
\text { even if you substantially contributed to the work? }\end{array}$} \\
\hline Yes & 16 & 41 \\
\hline No & 23 & 59 \\
\hline \multicolumn{3}{|c|}{ Have you been a recipient of gift authorship? } \\
\hline Yes & 23 & 59 \\
\hline No & 16 & 41 \\
\hline \multicolumn{3}{|c|}{ Has your rank been wrongly stated on an article? } \\
\hline Yes & 16 & 41 \\
\hline No & 23 & 59 \\
\hline \multicolumn{3}{|c|}{$\begin{array}{l}\text { Have you participated in a decision to grant } \\
\text { gift authorship? }\end{array}$} \\
\hline Yes & 19 & 49 \\
\hline No & 16 & 41 \\
\hline No answer & 4 & 10 \\
\hline
\end{tabular}

the study focused on specific clinical programmes. The decision to stress specific studies, as well as the wide experience of these senior researchers, assured our data of extra validity. The information obtained (tables 4-6) was based on experience and called for tact and openness during the interviews. Some resistance was noted at the beginning of the interviews and some participants were afraid that we would embarrass them. The length and quality of the interviews showed that this problem was easily overcome. The behaviour of the interviewees showed that they had begun to think about their publication practices, maybe for the first time.

\section{Ignorance of the ICMJE criteria}

As shown in a British study, ${ }^{1}$ ICMJE criteria are ignored by researchers. Bhopal et al showed that scientists agreed with the three criteria for authorship after these were explained. The French researchers agreed fully with the first two (substantial contribution to conception and design, or acquisition of data, or analysis and interpretation of data; drafting the article or revising it critically for important intellectual content), but had mixed views about the third (final approval of the version to be published). Their views on this last criterion could be questioned in view of the high frequency of ghost and gift authorship. As found in other studies, meeting of the three ICMJE criteria was rejected; ${ }^{1}{ }^{9}$ these criteria are viewed as being too strict to apply. The frequency of gift and ghost authorship was higher than observed in previous studies. ${ }^{2}{ }^{2}$ We were impressed by the openness of the participants, recognising that a majority of them had experienced gift authorship (table 6). The hierarchical structure common in French universities may explain this finding. We interviewed heads of department who wanted to be included as an author on any article from their department. It would have been interesting to interview young researchers on these practices. Behaviour between the generations is changing in clinical practice but is it changing in authorship practice?

\section{How can this ignorance of good practice in authorship be explained?}

We were surprised by the high frequency of gift and ghost authorship. These practices seemed quite common; few people question them and they are seen as normal in most cases. It is a serious problem, about which there is very little ethical discussion. The behaviour of researchers is usually very ethical; they apply good methodological practice in their scientific activities. However, when they discuss authorship and decide on the names that are to appear on an article, no guidelines are followed. They have no precise criteria for authorship and, if any criteria are used, these are decided within individual teams. Rules for authorship are insufficiently taught in France. There are many hypotheses to explain this behaviour: power, egoistic satisfaction, prestige, liberty, the "publish or perish" syndrome, and the evaluation of job candidates according to the number of their publications rather than quality.

\section{How can authorship understanding be improved in France?}

This situation has a negative impact on many research teams in medicine, and is responsible for much conflict and hard feelings that last for life. We do not know the real impact on academic promotion. The translation and adaptation of English guidelines will not be the best way to improve the situation in France. Such guidelines may be rejected and the implementation of any rules would then be delayed. Guidelines on authorship should be prepared by professionals. Three bodies could legitimately cooperate in this process: (1) learned societies, which have a mission to promote research; (2) representatives of biomedical journals; and (3) public research institutes and national agencies. It will take time to achieve success and the behaviour of scientists will have to change. It could take a generation to improve authorship practice in France.

\section{ACKNOWLEDGEMENTS}

We thank L Collet, MD, Chairman of the Scientific Committee, Hospices Civils de Lyon, for his helpful comments and for facilitating the interviews. We thank $M \mathrm{O}^{\prime}$ Connor, London, for copy-editing the paper, and O Gelpi, Director, Delegate for Research, Hospices Civils de Lyon, for identifying researchers, and all who agreed to answer our questions.

\section{Authors' affiliations}

B Pignatelli, F Chapuis, Public Health Department, Hospices Civils de Lyon, Lyon, France

H Maisonneuve, Public Health Department, Groupe Hospitalier

Lariboisière Fernand Widal, Paris University, Paris, France

\section{Competing interests: none declared}

BP, HM, and FC conceived and implemented the study; BP conducted the interviews and data analysis; $\mathrm{HM}$ and $\mathrm{BP}$ wrote the article; $\mathrm{FC}$ is guarantor of the article.

\section{REFERENCES}

1 Bhopal R, Rankin J, McColl E, et al. The vexed question of authorship: views of researchers in a British medical faculty. BMJ 1997;314:1009-12.

2 Rennie D, Flanagin A. Authorship! Authorship! Guests, ghosts, grafters, and the two-sided coin. JAMA 1994;271:469-71.

3 Flanagin A, Carey LA, Fontanarosa PB, et al. Prevalence of articles with honorary authors and ghost authors in peer-reviewed medical journals. JAMA 1998;280:222-4

4 Rennie D, Flanagin A, Yank V. The contributions of authors. JAMA 2000;284:89-91.

5 Rennie D, Flanagin A, Smith R, et al. Fifth international congress on peer review and biomedical publication. Call for research. JAMA 2003;289:1438.

6 Whenhui L, Shouchu Q, Yue Q. Authorship of published medical papers in three Chinese medical journals [Poster presented 2001 Sept 15]. Proceedings of the 4th International Congress on Peer Review in Biomedical Publication; 2001 Sept 14-16; Barcelona. http://www.ama-assn.org/public/peer/ prc_program2001.htm\#POSTERS (accessed 28 Feb 2005).

7 Reyes H, Jacard M, Herskovic V. Authorship in a medical journal from a developing country [Poster presented 2001 Sept 16]. Proceedings of the 4th International Congress on Peer Review in Biomedical Publication; 2001 Sept 14-16; Barcelona. http://www.ama-assn.org/public/peer/ prc_program2001.htm\#POSTERS (accessed 28 Feb 2005)

8 Paneth N. Separating authorship responsibility and authorship credit: proposal for biomedical journals. Reader response to authorship. Am J Public Health 1998; 88:824-6.

9 Hoen WP, Walvoort $\mathrm{HC}$, Overbeke AJ. What are the factors determining authorship and the order of authors' names? JAMA 1998;280:217-8. 